\title{
Wahrheit und Wahrhaftigkeit als medienethi- sches Thema im Zeitalter der Digitalisierung
}

\author{
»That is the real news « - Der Diskurs über Wahrheit in poli- \\ tischen Zusammenhängen als medienethisches Problem
}

Gotlind Ulshöfer

Real news sind seit Juli 2017 eine von dem Wahlkampfteam für Donald Trumps Wiederwahl 2020 selbst produzierte Sendung, die Nachrichten über den derzeitigen US-Präsidenten präsentiert, und auf verschiedenen Social-Media-Plattformen zu finden ist, u.a. auch bei Facebook. ${ }^{1}$ Die Schwiegertochter das Präsidenten, Lara Trump, ist als Nachrichtensprecherin dort mit den Worten zu hören "That is the real news $\mathbb{2}^{2}$. Die Sendung ist wie eine Nachrichtensendung inszeniert, obwohl die Filmclips eher den Eindruck von Wahlkampfspots machen, bei denen der Präsident und dessen Politik im Mittelpunkt stehen.

Am Beispiel von Real news lassen sich drei Problembereiche des aktuellen Umgangs mit dem Thema "Wahrheit " in Bezug auf Politik aufzeigen, die nicht nur ein medienethisches Problem darstellen, aber in diesem Artikel unter medienethischer Perspektive analysiert werden sollen. Erstens sind Erklärungen, dass eine Information über politische Ereignisse wahr ist, umstritten und werden selbst zu einem Politikum, bei dem der Rückbezug zur Wahrheit relativ unbegründet postuliert bzw. bestritten werden kann. Dies zeigt sich auch in der Bezeichnung der Sendung als "real news", die in Abgrenzung gegenüber "fake news" geschieht, mit denen vonseiten Donald Trumps Nachrichtensendungen etablierter Fernsehsender wie CNN bezeichnet werden. Aus medienethischer Perspektive ist hierbei die Frage nach Wahrheit zu verbinden mit der Analyse von Machtverhältnissen und damit auch der Frage nach der Definitionsmacht.

\footnotetext{
1 Vgl. Jonathan Lemire, Trump Daughter-in-Law Becomes Face of Re-Election Effort, in: The Washington Post 19. Oktober 2017, https://www.washingtonpost.com/national/trump-daughter-in-law-becomes-face-of-re-election-effort/2017/10/19/2685feca-b52d-11e7-9b93-b97043e57a22_story. html?utm_term=.39af269aa1d4 (20.10.2017).

2 Nachzusehen bspw. unter https://www.facebook.com/DonaldTrump/videos/vb. 153080620724/10160027734720725/?type=2\&theater $(20.10 .2017)$.
} 
Damit zusammen hängt ein zweiter Aspekt - die medialen Akteure: Die sogenannten Nachrichten bzw. Neuigkeiten werden nicht über einen derTV-Sender in die Öffentlichkeit gebracht, sondern es werden Social-Media-Plattformen wie Facebook genutzt, die einen hohen Verbreitungsgrad aufweisen. Das heisst auch, dass sich die Sendung nicht mehr dem Hierarchiegefüge und den Standards von Sendeanstalten einzuordnen braucht. Hier zeigt sich eine der Herausforderungen für eine medienethische Analyse:Wie kann und soll eine Medienethik aussehen, die traditionell im Bereich journalistischer Ethik und theologischerseits christlicher Publizistik verankert war, und durch die Digitalisierung nun auch weitere medial tätige Akteure in den Blick zu nehmen hat?

Drittens weist das Beispiel auch auf eine der zentralen ethischen Probleme hinsichtlich der Diskussion um Wahrheitsverständnisse hin, die gerade im medialen Bereich relevant sind: Wie hängen Wahrheit und Wirklichkeit zusammen? Die Forderung und das Selbstverständnis journalistischer Arbeit "wahrhaft « zu berichten, ist eng verknüpft mit der Vorstellung dadurch auch eine klare Analyse von Wirklichkeit zu bieten. Aber um welches Wirklichkeitsverständnis handelt es sich dabei?

Zusammenfassend lässt sich zunächst konstatieren, dass auf einer rhetorischen und medialen Ebene im politischen Bereich die Bezugnahme zum Stichwort "Wahrheit» im Sinne von "real news" und "fake news" genutzt wird, um politische Meinungen zu forcieren und Wirklichkeit zu prägen. Medienethisch stellt dies eine Herausforderung dar, weil $\mathrm{zu}$ fragen ist, warum ein traditionelles Wahrheitsverständnis im Sinne von "Tatsachenwahrheiten «", denen zumindest Nachrichtenmedien verpflichtet sein sollten, in den Hintergrund treten kann. Im Folgenden soll aufgezeigt werden, wie diese Phänomene mit Entwicklungen hinsichtlich der Digitalisierung zusammenhängen.

\footnotetext{
3 So der Begriff von Hannah Arendt, mit dem sie Tatschen und Ereignisse bezeichnet, die Wirklichkeit und politische Zusammenhänge prägen und beeinflussen, vgl. Hannah Arendt, Wahrheit und Politik, in: Dies., Wahrheit und Lüge in der Politik. Zwei Essays, München 1972, 44-92, hier 48.
} 


\section{Die Pluralität der Wahrheitsverständnisse und Wirklichkeiten im digitalen Zeitalter}

\section{Wahrheit als zentrales journalistisches Leitprinzip und ihre gesellschaftliche Problematisierung}

Im Journalismus, als dem klassischen Bereich der Medienethik, wird Wahrheit als grundlegendes Leitprinzip verstanden, das sich auf die Arbeitsweise der Journalistinnen und Journalisten, auf deren Arbeitsergebnisse und auch auf deren berufsethische Haltung bezieht. Der Schweizer Presserat schreibt beispielsweise in seiner Erklärung der Pflichten und Rechte der Journalistinnen und Journalisten im ersten Paragraphen über deren Pflichten : "Sie halten sich an die Wahrheit ohne Rücksicht auf die sich daraus für sie ergebenden Folgen und lassen sich vom Recht der Öffentlichkeit leiten, die Wahrheit zu erfahren. ${ }^{4}$ Gleichzeitig wird angemahnt, entsprechend seriös journalistisch zu arbeiten und dies bedeutet, orientiert an der Wahrhaftigkeit. Dabei wird mit Wahrhaftigkeit der Akt des Wahrheitsagens und damit auch eine journalistische Haltung gekennzeichnet. Diesen Pflichten werden Rechte gegenübergestellt, die unter anderem implizieren, dass die Journalistinnen und Journalisten ihrer Arbeit in Freiheit nachgehen können.

Das hier vorliegende Wahrheitsverständnis erinnert an Hannah Arendts Verständnis von "Tatsachenwahrheit « ${ }^{5}$ und geht gleichzeitig darüber hinaus. Auf epistemologischer Ebene wird bei dem hier vorgestellten Wahrheitsverständnis angenommen, dass es so etwas wie Wahrheit geben kann, die nachvollziehbar und an Fakten orientiert ist. Gleichzeitig steht Wahrheit in Relation zur Freiheit, die benötigt wird, um zur Wahrheit gelangen zu können. Diese Wahrheit wird über eine Tatsachenwahrheit hinaus gleichgesetzt mit Wahrhaftigkeit als einer Haltung, die Ausdruck einer bestimmten beruflichen Rolle ist. Dieser Zusammenhang führt dann auch dazu, dass das Zusammenspiel von wahrer Berichterstattung - im Sinne von sachlich angemessener -, die von einem dementsprechend handelnden Journalisten bzw. einer Journalistin ausgeführt wird, als Qualitätsmerkmal für "guten Journalismus" verstanden wird. ${ }^{6}$ Und

4 Schweizer Presserat, Erklärung der Pflichten der Journalistinnen und Journalisten, https://presserat.ch/journalistenkodex/erklaerungen/ (02.10.2017).

5 Arendt, Wahrheit und Politik, 57.

6 Vgl. bspw. den Sammelband:Wolfgang Wunden (Hg.), Wahrheit als Medienqualität. Beiträge zur Medienethik, Bd. 3, Frankfurt a.M. 1996. 
auch hier, in der Erklärung des Presserats, zeigt sich, dass journalistische Arbeit in ein Setting eingebunden ist, das in Relation steht mit Machtverhältnissen und gesetzlichen Regelungen. ${ }^{7}$ Obwohl es sich hierbei um "berufsethische Normen ${ }^{8}$ handelt, die sich auf Berufsjournalistinnen und -journalisten beziehen, ist das in der Schweizer Erklärung aufgezeigte Wahrheitsverständnis exemplarisch für den Bereich der Berichterstattung in vielen Ländern. ${ }^{9}$

Das darin zugrundeliegende Wahrheitsverständnis wird jedoch von mehreren Seiten in gegenwärtigen öffentlichen Diskussionen problematisiert. Erstens aus einer erkenntnistheoretischen Perspektive, die sich am Konstruktivismus orientiert und hierbei die Möglichkeit in Frage stellt, dass es so etwas wie objektive Wahrheit auch im journalistischen Bereich überhaupt geben kann. ${ }^{10}$ Und zweitens aus einer gesellschaftlich-politischen Lage, die hier exemplarisch an der Bundesrepublik Deutschland aufgezeigt sei, in der Teile der Bevölkerung den Wahrheitsgehalt von Nachrichten der traditionellen Medien, wie bundesweit erscheinende Tageszeitungen oder Berichte und Nachrichten gerade auch der öffentlich-rechtlichen Rundfunk- und Fernsehanstalten, anzweifeln: »Etwa ein Fünftel der Bevölkerung nutzt und vertraut sihren eigenen Internetquellen; das Vertrauen in traditionelle Medien, auch Qualitätsmedien, ist in den letzten Jahren nochmals gefallen, immerhin ein Fünftel der Bevölkerung hält den Begriff `Lügenpresse` für gerechtfertigt. «"

Dies lässt sich auch an der Bedeutung, die die Bezeichnung von "postfaktisch" und die Diskussionen um "fake news« bekommen hat, ablesen. Bei »fake news« werden gezielt Falschmeldungen eingesetzt, die aber oft auch in Form von Täuschung auftauchen: »Unter dem Deckmantel seriöser Berichterstattung veröffentlichen beispielsweise Rechtsextremistinnen und -extremisten Meldungen, die frei erfunden sind, oder sie basieren auf Nachrichten seriöser Medien, die verfälscht und mit rassistischen und demokratiefeindlichen

\footnotetext{
7 Vgl. bspw. Schweizer Presserat, Erklärung der Rechte der Journalistinnen und Journalisten,Absatz d, https://presserat.ch/journalistenkodex/erklaerungen/(02.10.2017). 8 Vgl. ebd.

9 Vgl. bspw. auch Deutscher Presserat, Publizistische Grundsätze, http://www.presserat.de/fileadmin/user_upload/Downloads_Dateien/Pressekodex2017light_web. pdf (02.10.2017).

${ }^{10} \mathrm{Vgl}$. Günter Bentele, Art. Wahrheit, in: Handbuch Medien- und Informationsethik, hg. v. Jessica Heesen, Stuttgart 2016, 59-66, hier 59. Zum Konstruktivismus auch: Andreas Klein/Ulrich H.J. Körtner, Die Wirklichkeit als Interpretationskonstrukt? Herausforderungen konstruktivistischer Ansätze für die Theologie, NeukirchenVluyn 2011.

${ }^{11}$ Bentele, Art. Wahrheit, 64.
} 
Untertönen versehen sind. ${ }^{12}$ Drittens wird Wahrheit als tragendes Element einer gesellschaftlichen Kommunikationsstruktur immer weiter relativiert, weil "die Macht von Emotionen und Effekten « ${ }^{13}$ wichtiger geworden ist, in deren Mittelpunkt nicht das Interesse an rational nachvollziehbarer Wahrheit steht.

Was ist der Unterschied an dieser Situation zu der Annahme, dass die Relativierung von Wahrheit schon immer Teil von Politik bzw. politischer Arbeit ist? Hannah Arendts Analyse zu »Wahrheit und Politik « ${ }^{14}$ postuliert, dass es - um Wahrheit aufzeigen zu können nötig ist, sich ausserhalb des politischen Bereichs zu stellen. ${ }^{15}$ Für sie ist der Raum des Politischen derjenige, in dem gemeinsam gehandelt wird und der aber auch begrenzt ist. Diese Begrenzung liegt für sie in den Dingen, »die Menschen nicht ändern können und die nur durch lügenden Selbstbetrug zum zeitweiligen Verschwinden gebracht werden können. «16 Tatsachen können zwar »umgelogen" werden, aber: »das klarste Zeichen der Faktizität eines Faktums ist eben dies hartnäckige Da « ${ }^{17}$. Ausserdem geht Arendt davon aus, dass "Macht ihrem Wesen nach niemals imstande ist, einen Ersatz für die Sicherheit und Stabilität der tatsächlichen Wirklichkeit zu bieten, die in der Vergangenheit wurzelt, als einer Dimension, die unserem Zugriff prinzipiell entzogen ist. « ${ }^{18}$ Für Arendt ist aber nur im Gegenüber der Politik und damit ausserhalb des politischen Bereichs Unparteilichkeit möglich. Diese schreibt sie dem Berichterstatter genauso zu wie dem Wissenschaftler bzw. der Universität und dem Richter.

Ausgehend von Arendts Überlegungen zeigt sich im Vergleich mit der gegenwärtigen Situation folgendes: Es scheint aufgrund von Digitalisierung und ubiquitär vorhandenem Zugang zu sozialen Medien nicht mehr so leicht zu sein, einen "politischen Bereich" getrennt zu analysieren vom persönlichen, ökonomischen etc. Dies führt auch dazu, dass verschiedene Formen von Wahrheit vermehrt vorhanden sind und teilweise auch miteinander in Konkurrenz stehen, so dass Aspekte wie persönliche "Lebenswahrheit» als ein "Authentischwerden des eigenen Lebens in seinen aktiven Vollzügen,

\footnotetext{
${ }^{12}$ Christiane Yavuz, Like - Share - Hate. Extremistische Umtriebe im Netz, in: merz. medien + erziehung 3 (2017), 13-19, hier 16.

${ }^{13}$ Ulrich H.J. Körtner, Für dieVernunft.Wider Moralisierung und Emotionalisierung in Politik und Kirche, Leipzig 2017, 9.

${ }^{14}$ Vgl. Arendt, Wahrheit und Politik, 44-92.

15 Vgl. ebd., 86.

${ }^{16}$ Ebd., 92.

17 Ebd., 84.

${ }^{18}$ Ebd., 85.
} 
seinen diese konstitutiv umgreifenden sozialen Bezügen und seiner leiblich-biographischen Bedingt- und Gewordenheit" und die "Wirklichkeitswahrheit", die die Lebenswahrheit insofern erweitert, weil sie diese in den Kontext von Wirklichkeit setzt und das Selbst herausfordert sich in der zu deutenden Wirklichkeit zu verorten, ineinander übergehen. ${ }^{19}$ Diese können dann auch im politischen Bereich relevant werden, weil sie medial über social media öffentlich gemacht werden können.

\section{Digitalisierung und die Schaffung von Wirklichkeiten}

Im Folgenden soll aufgezeigt werden, dass Digitalisierungsprozesse insofern für die Veränderungen von Kommunikationsstrukturen und auch Wahrheitsverständnissen mit verantwortlich sind, als hier die Möglichkeit besteht, eigene Wirklichkeiten zu schaffen und diese öffentlich zu machen bzw. mit anderen eine bestimmte Form von Öffentlichkeit zu teilen, wie sich beispielsweise bei der Formierung von Filterblasen ${ }^{20}$ zeigt.

Wie schon angemerkt, treten durch Digitalisierungsprozesse die Erhöhung der Zahl der Akteure in den Blick, die medial Wirklichkeit gestalten. Dabei gilt, dass gerade soziale Medien es ermöglichen, dass jeder und jede zum "Sender" bzw. der "Senderin" von Nachrichten, Daten, Berichten etc. wird. Ein definierter journalistischer Bereich ist zwar durch Medieninstitutionen wie Fernseh- und Radiosender und Zeitungen sowie Zeitschriften vorhanden, aber durch das Internet hat sich der Radius derjenigen, die nicht Berufsjournalist_innen sind und trotzdem publizieren, weit über ihren familiären Bereich und Freundeskreis hin zu einer globalen Öffentlichkeit ausgedehnt. Die Akteure, die dadurch Wirklichkeit medial gestalten, werden vielfältiger und finden sich auch in neuen Formen zusammen. So tragen Arten von Kollektivität wie »digitale Schwärme« auch zu einem Wandel des Öffentlichkeitsverständnisses bei und ermöglichen neue Formen des politischen Engagements und der Wirklichkeitsgestaltung. ${ }^{21}$ Gleichzeitig sind sie aber inhaltlich

\footnotetext{
${ }^{19}$ Das Zitat und dieser Abschnitt bezieht sich auf: Peter Dabrock, "Was heißt die Wahrheit sagen in fundamentaltheologischer Perspektive, in: Wahrheit. Marburger Jahrbuch Theologie XXI, hg. v. Wilfried Härle/Reiner Preul, Leipzig 2009, 91-130, hier $116 f$.

${ }^{20}$ Vgl. Eli Pariser, Filter Bubble. Wie wir im Netz entmündigt werden, München 2012.

${ }^{21}$ Vgl. Jens Kersten: Schwarmdemokratie. Der digitale Wandel des liberalen Verfassungsstaats, Tübingen 2017, 134-135.
} 
und technisch manipulierbar, bspw. durch "Social Bots". Diese "sind Computerprogramme, die eine menschliche Identität vortäuschen und darauf ausgerichtet sind, in sozialen Netzwerken wie Facebook und Twitter maschinell erstellte Beiträge wie Kommentare, Antworten oder Meinungsäußerungen zu generieren, um Diskurse zu beeinflussen bzw. zu manipulieren. ${ }^{22}$ Es können sich also auch durch technische Instrumentarien wie »Social Bots« Wirklichkeitsinterpretationen entwickeln, die zu Wahrheitsverzerrungen führen. Jenseits dieser gezielten Manipulationen und Beeinflussung von Wirklichkeitswahrnehmungen ergeben sich durch die Möglichkeiten der massiven Datenauswertungen, die unter dem Stichwort "Big Data « ${ }^{23}$ diskutiert werden, Interpretationen der Wirklichkeit, die wiederum selbst der Reflektion und theoretischen Auswertung bedürfen, um im Sinne einer Korrespondenztheorie zu wahrheitsrelevanten Fakten werden zu können. Ausserdem zeigt sich in der Nutzung von Algorithmen, ${ }^{24}$ wie sie beispielsweise beim Online-Handel oder in Suchmaschinen eingesetzt werden, eine Strukturierung von Wirklichkeit, die auf einer ersten Ebene von einer Maschine vermittelt wird und dabei auch die Perspektiven von "Wahrheit" relativieren. Trotz der technischen Instrumentarien ist jedoch wichtig festzustellen, dass Menschen letztendlich über Einsatz und Programmierung von "Social Bots" und Algorithmen verfügen und damit auch Menschen verantwortlich sind.

Daher führen Digitalisierungsprozesse zwar dazu, dass Wirklichkeiten vielgestaltiger werden und immer wieder neu konstituiert werden, aber diese Prozesse sind nicht völlig von menschlichen Aktivitäten zu trennen.

\section{Die Pluralität der kontextuellen Bezüge und Wahrheitsver- ständnisse im Hinblick auf Selbstverständnis und Analysen in der Medienethik}

Die Herausforderungen, die sich für die Medienethik aufgrund der Pluralität von Wirklichkeits- und Wahrheitsverständnissen ergeben, lassen sich nun genauer fassen und betreffen drei Dimensionen:

\footnotetext{
${ }^{22}$ Swenja Wütscher, Stichwort Social Bots, in: merz. medien + erziehung 3 (2017), 4.

${ }^{23}$ Vgl. Ramón Reichert, Big Data. Analysen zum digitalen Wandel von Wissen, Macht und Ökonomie, Bielefeld 2014.

${ }^{24}$ Vgl. Mercedes Bunz, Die stille Revolution. Wie Algorithmen Wissen, Arbeit, Öffentlichkeit und Politik verändern, ohne dabei viel Lärm zu machen, Berlin 2012.
} 
Zum Ersten geht es um das Selbstverständnis von Medienethik, das sich angesichts der Digitalisierung verändert und ausgeweitet hat. Wie sich exemplarisch am Beispiel der Entwicklung von Wahrheitsverständnissen zeigt, ist Medienethik nicht mehr allein auf journalistische Ethik zu reduzieren, sondern bedarf der Reflexion über digitale Entwicklungen auch im informationsethischen Bereich. Daher kann mit Jessica Heesen davon ausgegangen werden, dass Medien- und Informationsethik in einander übergehen - und sich beschäftigen mit »der Bewertung und Steuerung individuellen, gesellschaftlichen und institutionellen Handelns für eine sozialverträgliche Gestaltung von Informations- und Kommunikationstechniken wie auch mit der Verantwortung des und der Einzelnen bei ihrer Entwicklung,Verbreitung und Anwendung ${ }^{25}$.

Zum Zweiten liegt in der Vielzahl von Wirklichkeitsformen die Herausforderung, ob sich dadurch Medienwirklichkeit überhaupt analysieren lässt und was diese bedeutet. Meines Erachtens geht es auch in einer digital geprägten Gesellschaft bei der Frage nach der Beschreibung von Wirklichkeiten, die durch Medien vermittelt werden, darum, diese auf ihre Tatsachen-, Vernunft- und Lebenswahrheiten zu analysieren, wie oben ausgeführt. Hier ist auch Günter Benteles Ansatz, der zwar schon aus dem Jahr 1996 stammt, immer noch von Relevanz, der davon ausgeht, dass es bei einer MedienAnalyse darum geht, wie medial »andere Wirklichkeiten repräsentiert werden bzw. verzerrt werden. Gerade die Nicht-Beliebigkeit der Konstruktion, gerade der permanente Versuch der Herstellung adäquater (objektiver) und damit brauchbarer Medienwirklichkeiten ist die Hauptleistung der Medien [...]. Medienwirklich ist wirklich in einem buchstäblichen Sinne, im Sinn von wirkend. $\star^{26}$

Als dritte Herausforderung lässt sich formulieren, dass sich die medialen Wirklichkeiten nicht mehr allein durch das gedruckte Wort, sondern vor allem auch durch animierte Bilder aufzeigen. Ein zentraler Aspekt im Hinblick auf die Veränderung von Wirklichkeiten und damit auch von dem Verständnis von Wahrheit bzw. Wahrheiten in Relation zu verschiedenen Wirklichkeiten ist die, insbesondere durch Bilder vermittelte Konzentration auf das Affekthafte der Kommunikation im Netz. "Die digitalen Objekte

\footnotetext{
${ }^{25}$ Jessica Heesen, Einleitung, in: Handbuch Medien- und Informationsethik, hg. v. Ders., Stuttgart 2016, 1-9, hier 3.

${ }^{26}$ Günter Bentele, Wie wirklich ist die Medienwirklichkeit? Anmerkungen zum Konstruktivismus und Realismus in der Kommunikationswissenschaft, in: Wahrheit als Medienqualität. Beiträge zur Medienethik Band 3, hg. v. Wolfgang Wunden, Frankfurt am Main 1996, 121-142, hier 138.
} 
[...] haben zu großen Teilen keinen bloß kognitiven, sondern einen narrativen, ästhetischen, gestalterischen oder ludischen Charakter ${ }^{27}$. Andreas Reckwitz konstatiert diesbezüglich, dass das Bild "nur sekundär einen Informations-, primär hingegen einen Affektcharakter, das heißt eine ästhetische oder narrative Form ${ }^{28}$ hat. Bezieht man diese Entwicklungen auf den Umgang mit Wahrheit und Wahrhaftigkeit so wird deutlich, warum diese in einer digital geprägten Welt eher in den Hintergrund zu treten scheinen. Der ludische Charakter der Kommunikation und der Informationsvermittlung, die zum Teil ja auch durch "Gamification $"^{29}$ geprägt ist, kann dazu führen, dass hierbei die Standards der Informationsübermittlung, für die journalistische Kodizes als exemplarisch gelten können, keine grosse Rolle mehr spielen. Elemente des Spielerischen, Ästhetischen und Künstlerischen werden prägend, in denen Freiräume jenseits von Tatsachenwahrheiten entstehen können, die wieder zu neuen Wirklichkeiten bzw. mindestens zur Veränderung von Wirklichkeitswahrnehmung führen können.

Angesichts der Veränderungen und Pluralisierungen von Wahrnehmung und Schaffung von Wirklichkeiten stellt sich die Frage, wie in diesem Kontext und aus einer ethischen Perspektive von Wahrheit und Wahrhaftigkeit gesprochen werden kann: Ist Wahrheit ein ethisches Kriterium, wie es sich im Berufskodex für Journalistinnen und Journalisten zeigt? Geht es gerade angesichts der verschiedenen Ebenen von Wirklichkeitsanalysen und -schaffung darum, dass Wahrheit etwas ist, was Wirklichkeit zu erschliessen hilft, worauf Dietmar Mieth aufmerksam macht: "Wahrheit ist, was sprachlich, sachlich, praktisch Wirklichkeit erschließt «. ${ }^{30}$ Oder gilt es aufgrund der Vielschichtigkeit von Wirklichkeiten und Wahrheiten dem Wahrheitsbegriff nur wenig Relevanz einzuräumen?

\footnotetext{
27 Andreas Reckwitz, Die Gesellschaft der Singularitäten. Zum Strukturwandel der Moderne, Berlin 2017, 235.

${ }^{28}$ Ebd.

${ }^{29}$ Vgl. Susanne Strahringer/Christian Leyh (Hg.), Gamification und Serious Games. Grundlagen,Vorgehen und Anwendungen, Wiesbaden 2017.

${ }^{30}$ Dietmar Mieth, Die neuen Tugenden. Ein ethischer Entwurf, Düsseldorf 1984, 160.
} 


\section{Was ist Wahrheit und was ist Wahrhaftigkeit für eine Medienethik im digitalen Zeitalter?}

\section{Fake News, Lüge, Täuschung, Halbwahrheiten im Kontext von Wahrheit als ethischer Leitorientierung - oder: „Wie wird mein Wort wahr? « ${ }^{31}$}

Wahrheit und Wahrhaftigkeit sind trotz der Pluralisierung von gesellschaftlichen Kontexten aufgrund der Digitalisierung und damit auch trotz der eigenen Pluralisierung von Wahrheitsverständnissen gerade hinsichtlich einer Tatsachenwahrheit auch für eine Medienethik als Orientierungsfaktor nicht zu verabschieden. Gleichzeitig zeigt sich, dass ein ethisch fundiertes Wahrheitsverständnis angesichts der Vielzahl der Wirklichkeitsverständnisse und der Möglichkeiten, Wahrheit zu verstehen, kein rigoristisches Verständnis eines absolut $\mathrm{zu}$ setzenden, puristischen Wahrheitsverständnisses sein kann. Es geht darum, ein situativ angemessenes Wahrheitsverständnis zu entwickeln, das dabei helfen kann, gerade auch im medialen Bereich mit Wahrheit und Lüge und ihren weiteren Ausprägungen umzugehen. Anregungen für ein in diesem Sinne differenziertes Verständnis von Wahrheit sind in Dietrich Bonhoeffers fragmentarischem Aufsatz Was heißt die Wahrheit sagen? ?32 $^{3 \mathrm{zu}}$ finden. Er macht dort deutlich, dass es bei der Frage nach der Wahrheit und bei der Beantwortung der Frage, "Wie wird mein Wort wahr?", um den Anlass des Sprechens, um den Standort sowie um den Inhalt geht, oder wie Bonhoeffer formuliert: »indem ich den Gegenstand, über den ich etwas aussage, in diesen Zusammenhang bringe " $^{33}$.

Daher will ich im Folgenden Bonhoeffers Analyse einer »lebendigen Wahrheit ${ }^{34}$, die in Relation zur Situation steht, und dabei dennoch in einem christlich-theologischen Weltverständnis verankert ist, aufgreifen und überlegen, inwiefern sie bei der Suche nach Ant-

\footnotetext{
${ }^{31}$ Dietrich Bonhoeffer, Was heißt die Wahrheit sagen?, in: Ders., Konspiration und Haft 1940-1945, hg. v. Jørgen Glenthøj/Ulrich Kabitz/Wolf Krötke, Dietrich Bonhoeffer Werke, Bd. 16, Gütersloh 1996, 619-629, hier 628.

${ }^{32}$ Ebd., 619-629.

${ }^{33}$ Ebd., 628.

${ }^{34}$ Vgl. Christiane Tietz-Steiding, Lebendige Wahrheit - wirklichkeitsgemäße Lüge. $\mathrm{Zu}$ einem Aufsatzfragment Dietrich Bonhoeffers, in: Oliver Hochadel/Ursula Kocher (Hg.), Lügen und Betrügen. Das Falsche in der Geschichte von der Antike bis zur Moderne, Köln u.a. 2000, 273-282.
} 
worten hinsichtlich der ethischen Beurteilung von Wahrheitsfragen im Kontext von digitalen Wirklichkeiten weiterhilft.

$\mathrm{Zu}$ diesem Verständnis gehört auch, dass Wahrheit nicht absolut zu setzen ist, sondern in einer doppelten Ausrichtung steht, nämlich in Relation zu dem lebendigen Gott und in Relation zur Situation. »Darum geht es ja gerade, wie ich die wahrheitsgemäße Rede, die ich Gott schulde, in meinem konkreten Leben mit seinen mannigfaltigen Verhältnissen, zur Geltung bringe. Die Gott geschuldete Wahrheitsgemäßheit unserer Worte muß in der Welt konkrete Gestalt annehmen. Unser Wort soll nicht prinzipiell, sondern konkret wahrheitsgemäß sein. Eine nicht konkrete Wahrheitsgemäßheit ist vor Gott gar nicht wahrheitsgemäß $\ll^{35}$. Theologisch bleibt der Anspruch auf Wahrheit in einem Gottesverständnis verankert, das davon ausgeht, dass Gott die Wahrheit ist, und insofern menschliche Wahrheit als ein innerweltliches Geschehen zu verstehen ist.

Mit Bonhoeffer lassen sich also bezüglich des Wahrheitsverständnisses folgende Ebenen der Verantwortungswahrnehmung in Bezug auf die Wahrhaftigkeit der Rede unterscheiden:

Erstens geht es um Wahrheit als Beziehungsgeschehen: Im Zentrum von Bonhoeffers Analyse zur Wahrheit steht der Sprechakt, der Beziehung schafft und Ausdruck von Beziehung ist. Dieser Anlass des Sprechens und die Beziehung zum Gegenüber und zur Sache beeinflussen für ihn, wie Wahrheit in diesem Beziehungsgeschehen vermittelt werden soll. Betrachtet man Kommunikation in der digitalen Welt und hierbei insbesondere in den sozialen Medien, so fällt auf, dass es sich hierbei nicht allein um das Sprechen bzw. Schreiben handelt, das wesentlich ist, sondern dass sich ein Grossteil des Kommunikationsgeschehens über Bilder vermittelt und hierbei, wie wir gesehen haben, Affekte im Zentrum stehen.

Über Bonhoeffer hinausgehend gilt es daher zu überlegen, inwiefern Unterschiede zu suchen sind zwischen Sprechen, Schreiben und Bildern und deren Wahrheitsgehalten sowie der Frage, wie und an welchem Ort bzw. welcher Webseite oder welcher Social-MediaPlattform diese Beziehungsgeschehen vermittelt werden. Mit HansMartin Gutmann kann für die persönlichen Beziehungen tatsächlich eingefordert werden, dass es sich dort möglichst "um lügenfreie Zonen ${ }^{36}$ handeln sollte, weil diese beispielsweise besonders unter pädagogischen Gesichtspunkten wichtige Beziehungen darstellen,

\footnotetext{
35 Bonhoeffer, Wahrheit, 621.

${ }^{36}$ Hans-Martin Gutmann, Wir brauchen lügenfreie Zonen, in: Rochus Leonhardt/ Martin Rösel (Hg.), Dürfen wir lügen? Beiträge zu einem aktuellen Thema, Neukirchen-Vluyn 2002, 140-157, hier 156.
} 
in denen Kindern Wahrheit vermittelt werden kann. So macht er deutlich, dass »Lügen in intimen Beziehungen immer zerstörerisch wirkt, während es für die Durchsetzung ökonomischer und politischer Interessen bisweilen als nützlich angesehen wird - und medialvirtuelle Welten in vielen Aspekten gerade dadurch sfunktionieren', daß sie in Hinblick auf Wahrheit, Wahrhaftigkeit und Richtigkeit zumindest anderen Standards folgen ${ }^{37}$.

Hier kann sich die Frage anschliessen, inwiefern Wahrheit in einem Beziehungsgeschehen, das im Sinne einer "persönlichen Öffentlichkeit " $^{38}$ über eine Facebook-Seite vermittelt wird, zu einer grundlegenden Kategorie des Handelns und Darstellens werden sollte. Dabei scheint sich das Kriterium, dass persönliche Beziehungen der Wahrheit bzw. zumindest der Wahrhaftigkeit benötigen, um beständig sein zu können, zu relativieren. Gerade der Zwischenraum von nicht mehr ganz privat, aber nicht immer ganz öffentlich, den Facebook-Seiten darstellen können, sowie deren ökonomische Nutzung durch Facebook oder durch andere Unternehmen, die die Daten und bereitgestellten Informationen auswerten, lässt die Antwort auf die Frage nach der Relevanz der Wahrheit in diesem Kontext wiederum in einem Bonhoefferschen Sinne als relativ erscheinen. In diesem Sinne kann aus einer theologischen Perspektive die Bedeutung der Unterscheidung zwischen "Selbstdarstellungen von Facebook als Repräsentationen von der physischen Präsenz einer Person und deren lebendigem Selbstvollzug « ${ }^{39}$ wichtig werden. Wo Grenzen zu ziehen sind, zwischen einem spielerischen Verändern der eigenen Identität und einer "wahrhaftigen" Darstellung muss im Blick auf den jeweiligen Fall und hinsichtlich der Kriterien des Nicht-Schadens und der "Lebensdienlichkeit» entwickelt werden.

Wahrheit als Beziehungsgeschehen im Blick auf die breite Öffentlichkeit des Internets wird dann zur Herausforderung, wenn sich "Filterblasen « bilden und so "communities" entstehen, die ihre eigenen, sogenannten "Wahrheiten" propagieren. ${ }^{40}$ In diesen Überlegungen zeigt sich, dass Wahrheit als Beziehungsgeschehen allein noch kein ethisches Kriterium darstellt, sondern es noch weiterer Aspekte eines Wahrheitsverständnisses bedarf, damit Wahrheit in

\footnotetext{
${ }^{37}$ Ebd., 156.

${ }^{38}$ Jan Schmidt, Das neue Netz: Merkmale, Praktiken und Folgen des Web 2.0, Kons$\operatorname{tanz} 2009,107$.

${ }^{39}$ Christina Ernst, Mein Gesicht zeig ich nicht auf Facebook. Social Media als Herausforderung theologischer Anthropologie, Göttingen 2015, 342.

${ }^{40}$ Pariser, Filter Bubble, 165.
} 
einem pluralen Kontext unter ethischen Gesichtspunkten relevant werden kann.

Zweitens:Wahrheit in Relation zur Freiheit im Kontext des Ortes und des Standpunkts: Meines Erachtens ist Bonhoeffers Analyse hier weiter, über den Nahbereich hinaus, zu entwickeln. Wie schon der journalistische Kodex deutlich macht, bedarf es zur Suche nach Wahrheit freiheitlicher Strukturen, die Zugang zu Informationen und Recherchen ermöglichen und in denen ungestraft wahrheitsgemässe Antworten gegeben werden können, um selbst wiederum einen Standpunkt, der Wahrheit impliziert, einnehmen zu können. Betrifft dieses Verhältnis von Wahrheit und Freiheit die Voraussetzungen zur Wahrheit zu gelangen, so kann Wahrheit auch Wirklichkeit erschliessen und in diesem Sinne Freiheit fördern, weil neue Perspektiven eröffnet werden, die die wahren Taten, die hinter einem Fall liegen können, aufdecken. Dies geht über Bonhoeffers Anliegen, dass ich den "Ort erkenne, an dem ich stehe ${ }^{41}$ hinaus - und ist aber auch für mediale Kontexte im Zeitalter der Digitalisierung von Bedeutung. Wahrheit im Sinne von Tatsachenwahrheit und Lebenswahrheit bedarf der Freiheit und trägt als Charakteristikum das Streben nach Freiheit in sich, das aber jeweils in Relation zum Kontext wirksam werden muss.

Drittens, Wahrheit als Anspruch auf Wirklichkeitsgemässheit: Hierbei geht es gerade aus einer theologischen Perspektive nicht allein um Fakten. Aufgrund des Wissens um die Heilstat Gottes, d.h., dass Jesus Christus zur Versöhnung in diese Welt gekommen ist und so Welt und Gott in enge Relationen setzt, d.h. »in ihm begegnen sich Gotteswirklichkeit und Weltwirklichkeit «"2 , wird das Verständnis von Wirklichkeit in einen neuen Horizont gesetzt. Die Pluralität der Wirklichkeiten, die mit medialer Vermittlung durch soziale Medien verstärkt wurde, führt dazu, dass es für das gesellschaftliche Miteinander um die Suche nach gemeinsamen Wirklichkeitsverständnissen gehen muss, in denen so etwas wie Wahrheit gefunden werden kann und in dem Wahrheit auch zum Kriterium der Angemessenheit werden kann. Dass dies ein Geschehen ist, in dem sowohl Machtansprüche als auch Konflikte zum Tragen kommen, ist in pluralen Gesellschaften unvermeidlich. ${ }^{43}$ Dass damit auch einhergeht, dass Wahrheitsansprüche inkommensurabel sein können,

\footnotetext{
${ }^{41}$ Bonhoeffer, Wahrheit, 628.

42 Tietz-Steiding, Lebendige Wahrheit, 279.

${ }^{43}$ Vgl. Heinzpeter Hempelmann, Faktisch, postfaktisch, postmodern? Kommunikation von Wahrheit(sansprüchen) in pluralistischen Gesellschaften als Problem und Herausforderung, in: theologische beiträge 48 (2017), 6-23, hier 16.
} 
sollte jedoch nicht dazu führen, dass die gemeinsame Suche nach Wahrheit aufgegeben wird. Aus einer theologischen Perspektive kann dazuhin beigetragen werden, dass Wahrheit-sagen nicht ohne Liebe geschehen sollte. ${ }^{44}$

Aber wie kann ein hier entwickeltes kontextuelles Verständnis von Wahrheit trotzdem ethisch relevant werden, ohne dass es in der Relativierung stecken bleibt?

\section{Wahrhaftigkeit als verantwortungsethisch orientierte Antwort angesichts der bleibenden Relevanz von Wahrheit}

Aus einer medienethischen Perspektive geht es nicht nur um Steuerungsfragen, wie Medien organisiert sein sollten. Wie wir gesehen haben, ergibt sich aus dem Phänomen der Ubiquität von Medien - in Form von digitalen Geräten und ihren Zugängen zum Internet und zu virtuellen Dimensionen -, dass sich Lebensbereiche verstärkt überschneiden. Hinsichtlich der Frage, wie hierbei medienethisch umgegangen werden sollte, ist m.E. zwar nicht nur auf einer individualethischen Ebene anzusetzen, aber im Folgenden soll diese in den Vordergrund treten, denn hier ergeben sich wichtige Ansatzpunkte. Gerade angesichts der Bedeutung, die individuelles Handeln in einer pluralisierten Gesellschaft immer noch hat, ist hierbei die Frage nach der Verantwortung zentral. Mit Bonhoeffer ist hier ein Verantwortungsverständnis stark zu machen, mit dem sowohl moralisch-ethische Fragen thematisiert werden können als auch Abwägungen vorgenommen werden können, was eine ethisch angemessene Aktion bzw. Reaktion ist. Das heisst auch: "Verantwortlich handeln bedeutet von hier aus, die menschliche Wirklichkeit, als von Gott in Christus angenommene in die Gestaltung des Handelns einbeziehen ${ }^{45}$. Es stellt sich jedoch hier die Frage, von welcher Wirklichkeit auszugehen ist. Ausgangspunkt zur Analyse von Wirklichkeit kann Wahrhaftigkeit sein. Wahrhaftigkeit soll dabei als »eine auf das handelnde Subjekt bezogene Norm verstanden [werden], möglichst wahrheitsgemäß zu kommunizieren. Wahrhaftigkeit bringt das Verhältnis des Menschen zur Wahrheit zum Ausdruck und bezeichnet das subjektive «Für-wahr-halten`von

\footnotetext{
${ }^{44}$ Vgl. Eberhard Schockenhoff, Zur Lüge verdammt? Politik, Medien, Medizin, Justiz, Wissenschaft und die Ethik der Wahrheit, Freiburg u.a. 2000, 122, der auf den Konflikt zwischen Wahrheit und Liebe aufmerksam macht.

${ }_{45}$ Bonhoeffer, Ethik, hg. v. Ilse Tödt/Heinz Eduard Tödt/Ernst Feil/Clifford Green, Dietrich Bonhoeffer Werke, Bd. 6, München 1992, 223.
} 
etwas ${ }^{46}$. Diese verantwortungsethische Orientierung, die Wahrhaftigkeit impliziert, mag zunächst naiv erscheinen.Aber Wahrhaftigkeit, die mit einem am Bonhoefferschen ausgerichteten Verständnis von Wahrheit verbunden wird, ermöglicht sowohl Analysen von Wirklichkeitsverständnissen im Hinblick auf ihr Wahrheitsverständnis als auch angemessene Aktionen bzw. Reaktionen bezüglich einer vorgegebenen oder zu gestaltenden Situation, die nicht nur reine Tatsachenwahrheit erfordert. Hierbei gilt es im Sinne der Wahrhaftigkeit, die sich in Genauigkeit und Ernsthaftigkeit ausdrückt, die verschiedenen Dimensionen von Wirklichkeit zu analysieren. ${ }^{47}$

Diese Haltung der Wahrhaftigkeit, die Teil eines Verantwortungsverständnisses ist, kann dann auch ihren Beitrag bei der Suche nach und dem Finden von angemessenen Regeln zur gesellschaftlichen Gestaltung von Medien sowie deren Nutzung und Produktion leisten. ${ }^{48}$

- Prof. Dr. Gotlind Ulshöfer ist Gastprofessorin für Theologische Ethik an der Theologischen Fakultät der Universität Zürich. Zu ihren Arbeitsgebieten gehören insbesondere Sozialethik und Grundlagenfragen der Ethik, Wirtschafts-, Unternehmens- und Finanzmarktethik, Ethik im Zeitalter der Digitalisierung und Prozesstheologie.

\footnotetext{
46 Bentele, Art. Wahrheit, 61.

${ }^{47}$ Vgl. zu diesen beiden Bestimmungen von Wahrheit: Bernard Williams, Wahrheit und Wahrhaftigkeit, Frankfurt a.M. 2003.

${ }^{48} \mathrm{Zu}$ diesen drei Aspekten vgl. Schockenhoff, Zur Lüge verdammt, 334ff.
} 